\title{
ЭФФЕКТИВНОСТЬ ОБНАРУЖЕНИЯ СИГНАЛА С НЕИЗВЕСТНЫМ НЕЭНЕРГЕТИЧЕСКИМ ПАРАМЕТРОМ С ИСПОЛЬЗОВАНИЕМ АЛГОРИТМОВ HА ОСНОВЕ ТЕОРИИ СОMРRESSIVE SENSING
}

\author{
Д. Ю. ГОЛОВАНОВ, В. И. ПАРФЕНОВ
}

Воронежский государственный университет, Россия, Воронеж, 394018, Университетская пл., д. 1

\begin{abstract}
Аннотация. В работе рассмотрена задача обнаружения квазидетерминированных сигналов на фоне шума при цифровой обработке. При этом в качестве критерия синтеза подобных алгоритмов использован критерий минимума затрачиваемых арифметических операций при заданной эффективности обнаружения. С этой целью подобные алгоритмы синтезированы на основе принципов теории Compressive Sensing. C целью проверки эффективности разработанных алгоритмов проведено их компьютерное моделирование, в результате которого определено, каким образом полная вероятность ошибки обнаружения зависит от отношения сигнал/шум и степени сжатия (отношение числа элементов в векторах достаточной статистики до и после «сжатия»). Определены потери в эффективности обнаружения предложенных алгоритмов по сравнению с классическим, оптимальным в соответствии с методом максимального правдоподобия, при различных значениях отношения сигнал/шум и степени сжатия. Одновременно, указан выигрыш в количестве используемых арифметических операций предложенных алгоритмов по сравнению с классическим. Приведены результаты, позволяющие сделать обоснованный выбор алгоритма обнаружения в зависимости от имеющихся аппаратных возможностей и допустимого ухудшения эффективности обнаружения.
\end{abstract}

Ключевые слова: алгоритм обнаружения; отношение правдоподобия; достаточная статистика; неэнергетический параметр; разреженный сигнал; полная вероятность ошибки; критерий идеального наблюдателя; отношение сигнал/шум; корреляционная функция; orthogonal matching pursuit; compressive sensing

\section{ВВЕДЕНИЕ}

Теория обнаружения является известным, хорошо разработанным вопросом статистической радиотехники, неоднократно излагавшимся в монографиях и учебной литературе [1-4]. Однако исследования по теории обнаружения продолжаются, о чем свидетельствуют регулярно появляющиеся журнальные статьи, посвященные этой теме.

Обычно рассматриваются только специальные вопросы этой теории. Данная статья посвящена разработке и исследованию новых алгоритмов обнаружения цифровых сигналов с неизвестными параметрами, при практиче- ской реализации которых требуется значительно меньшее количество математических операций по сравнению с известными.

В последние годы наметилась устойчивая тенденция к построению радиосистем различного назначения полностью в цифровой области [5]. При этом сигнал с выхода приемной антенны подается сразу в аналого-цифровой преобразователь и дальнейшая обработка осуществляется непосредственно над отсчетами входного сигнала. Количество обрабатываемых отсчетов определяется максимальной частотой в спектре этого сигнала $[4,5]$. Увеличение этой частоты, что также является тенден- 


\section{БИБЛИОГРАФИЧЕСКИЙ СПИСОК}

1. Хелстром, К. Статистическая теория обнаружения сигналов. Пер. с англ. Г. Ю.Кобзаревой, под ред. Ю.Б.Кобзарева. М.: Иностранная литература, 1963. 432 c.

2. Ван Трис, Г. Теория обнаружения, оценоки модуляции. Т. 1. Пер. с англ. под ред. В.И. Тихонова. М.: Сов. радио. 1972.744 с.

3. Акимов, П.С.; и др. Теория обнаружения сигналов. Под ред. П.А.Бакута. М.: Радио и связь, 1984. $440 \mathrm{c}$.

4. Шахтарин, Б.И. Обнаружение сигналов: учебное пособие для вузов. 3-е изд., испр. М.: Горячая линия-Телеком, 2015. $464 \mathrm{c}$.

5. Вишневский, В. М.; и др. Широкополосные беспроводные сети передачи информации. М.: Техносфеpa, 2005. $592 \mathrm{c}$.

6. Candes, E. J.; Wakin, M. B. "An introduction to compressive sampling," IEEE Signal Processing Mag., Vol. 25, No. 2, P. 21-30, 2008. DOI: 10.1109/MSP.2007. $\underline{914731}$.

7. Donoho, D. L. "Compressed sensing," IEEE Trans. Inf. Theory, Vol. 52, No. 4, P. 1289-1306, 2006. DOI: $10.1109 /$ TIT.2006.871582.

8. Eldar, Y. C.; Kutyniok, G. Compressed Sensing: Theory and Applications. Cambridge University Press, 2012. $555 \mathrm{p}$.

9. Foucart, S.; Rauhut, H. A Mathematical Introduction to Compressive Sensing. Springer, 2013. 625 p. DOI: 10.1007/978-0-8176-4948-7.

10. Парфенов, В. И.; Голованов, Д. Ю. “Определение незанятых частотных поддиапазонов по сжатым измерениям," Инфокоммуникационные технологии, T. 13, № 3, C. 305-312, 2015. DOI: 10.18469/ikt.2015. 13.3.11.

11. Парфенов, В.И.; Голованов, Д.Ю. “Принципы построения и анализ эффективности функционирования беспроводных сенсорных сетей на основе теории Compressive Sensing," Цифровая обработка сигналов, № 2, С. 14-19, 2016.

12. Парфенов, В.И.; Голованов, Д.Ю. “Обнаружение дискретных разреженных сигналов с частотой дискретизации, не превышающей частоту Найквиста,” Журнал радиоэлектроники, № 6, 2017. URI: http://jre.cplire.ru/jre/jun17/1/abstract.html.

13. Draganic, A.; Orovic, I.; Stankovic, S. "On some common compressive sensing recovery algorithms and applications," Facta universitatis - series: Electronics and Energetics, Vol. 30, No. 4, P. 477-510, 2017. DOI: 10.2298/FUEE1704477D.

14. Abo-Zahhad, M. M.; Hussein, A. I.; Mohamed, A. M. "Compressive sensing algorithms for signal processing applications: a survey," Int. J. Commun., Network System Sci., Vol. 8, No. 6, P. 197-216, 2015. DOI: 10.4236/ijens.2015.86021.

15. Allen-Zhu, Z.; Gelashvili, R.; Razenshteyn, I. "Restricted isometry property for general p-norms," IEEE Trans. Inf. Theory, Vol. 62, No. 10, P. 5839-5854, 2016. DOI: $10.1109 /$ TIT.2016.2598296. 
16. Стренг, Г. Линейная алгебра и ее применения. Пер. с англ. Ю.А.Кузнецова и Д.М.Фаге, под ред. Г.И.Марчука. М.: Мир, 1980. 456 с.

17. Tropp, J. A.; Gilbert, A. C. "Signal recovery from random measurements via orthogonal matching pursuit," IEEE Trans. Inf. Theory, Vol. 53, No. 12, P. 4655-4666, 2007. DOI: $10.1109 /$ TIT.2007.909108.

18. Cai, T. T.; Wang, L. "Orthogonal matching pursuit for sparse signal recovery with noise," IEEE Trans. Inf. Theory, Vol. 57, No. 7, P. 4680-4688, 2011. DOI: $10.1109 /$ TIT.2011.2146090.

19. Wang, J.; Shim, B. "Exact recovery of sparse signals using orthogonal matching pursuit: how many iterations do we need?," IEEE Trans. Signal Processing, Vol. 64, No. 16, P. 4194-4202, 2016. DOI: 10.1109/TSP. 2016.2568162 .
20. Needell, D.; Tropp, J. A. "CoSaMP: Iterative signal recovery from incomplete and inaccurate samples," Applied and Computational Harmonic Analysis, Vol. 26, No. 3, P. 301-321, 2009. DOI: 10.1016/j.acha.2008.07. $\underline{002}$.

21. Sturm, B. L.; Christensen, M. G. "Comparison of orthogonal matching pursuit implementations," Proc. of 20th European Signal Processing Conf., EUSIPCO, 27-31 Aug. 2012, Bucharest, Romania. IEEE, 2012, P. 220-224. URI: https://ieeexplore.ieee.org/document/6333943/.

22. Berinde, R.; Indyk, P.; Ruzic, M. "Practical near-optimal sparse recovery in the L1 norm," Proc. of 46th Annual Allerton Conf. on Communication, Control, and Computing, 23-26 Sept. 2008, Urbana-Champaign, IL, USA. IEEE, 2008, P. 198-205. DOI: 10.1109/ ALLERTON.2008.4797556.

После переработки 15.06.2018 